\title{
Intelligent System for Continuous Steel Casting Based on Water Flow Control in the Secondary Cooling Stage
}

\author{
Gelu-Ovidiu Tirian ${ }^{1}$, Ioan Filip ${ }^{2}$ and Cristian Paul Chioncel ${ }^{3}$ \\ ${ }^{1}$ Politehnica University of Timisoara/Department of Electrical Engineering and Industrial Informatics, Hunedoara, Romania \\ ${ }^{2}$ Politehnica University of Timisoara/Department of Automation and Applied Informatics, Timisoara, Romania \\ ${ }^{3}$ Eftimie Murgu University/Department of Electrical Engineering and Informatics, Resita, Romania
}

\begin{abstract}
This paper presents an intelligent system capable of eliminating the surface flaws and casting rejects, to increase the quality of continuously cast products and implicitly to increase the productivity of the installation. This method contains a fuzzy controller which requires changes of the water flow value at the secondary cooling. Fuzzy system controlling in real time the distribution of the water flow rate according to the real situation in the installation and working as an adaptive. The simulations results show us that the control strategy proposed is a very efficient method for the water flow control in the secondary cooling stage.
\end{abstract}

\section{Introduction}

Nowadays, continuous casting provides the biggest part of semi-products for the metallurgical industry. The process is a very complex one, of high productivity, but, because of some technological issues it can generate quality defects in the wire or throw-outs by cracking the crust of the semi-product. This defects and throw-outs lead to lower quality of the semi-products, of the installation productivity and to economic losses. The continuous casting domain has developed and consolidated as an important research direction, concentrating collective research efforts of numerous specialists from the academic domain and from the metallurgical industry. The method of continuous casting consists of introducing the liquid metal with a well determined temperature in a cava shape which has the walls cooled inside with water named crystallizing, and the evacuation is made on the opposite side where is obtained the solidified chord [1-3].

A very important component of the continuous casting installation is the secondary cooling zone. The secondary cooling zone has the role to continue the wire cooling after it has emerged from the crystallizing and to assure the total solidification of the semi-product. It is considered "the heart" of a continuous casting and has the role of ensuring the quality of the material, the material surface shape and has to ensure a homogeneous cooling and a uniform repartition of the water on the materials surface [4].

The crust created in the crystallizing gives the shape of the molded section. In most cases the crust doesn't offer sufficient mechanical resistance to the action of ferro static pressure $[5,6]$. To complete solidification and guidance in good conditions of the wire it is created the secondary cooling zone. This cooling is achieved by direct spraying pressurized water, through nozzles, it is able to cross the steam layer formed by evaporation and ensure continuous water-metal contact.

The secondary cooling can be made in different cooling environments. In practice, the water is used especially as cooling agent that is sprayed through nozzles (circular conical, conical ring and slot). In special cases it is added to the water compressed air for optimizing the automation sprayed water [7].

The heat from the wire is partially removed by heating the cooling water, but the most heat is extracted by water evaporation, even if the evaporation percentage of the sprayed water on the wires surface is in general lower than $20 \%$. All the unevaporated water which flows from the wires surface are collected filtered and reused.

The importance of the problem from the scientific point of view is reducing the casting defects, increasing the productivity and increasing the quality of the continuous casting semi - products, and from the technological point of view is very important to assure the cooling programs, all kind of casting speeds and temperatures on which the products are casted. The secondary cooling is important because of the process that creates the crust, it is leading to a length adjustment technology so that surface effects to be minimized [8].

At this moment the cooling is done using some valves by using some classical PID loops which adjust the water flow depending on reference measure. Advantages of PID control are a relatively easy implementation, in general only three parameters $\mathrm{P}, \mathrm{I}$, and $\mathrm{D}$ to be set up and an automatic parameter tuning mechanism. The main drawback of PID is, however, its non-convenience for the control of non-linear systems and its use in systems 
with fast changes of process parameters. In [9] is described and discussed challenges and control issues of the PID use for the continuous cast-ing and also is analyzed limitations of PID control. In comparison to PID control the fuzzy logic offers better control performance, smaller, overshoots, faster response, robustness and stability [7].

In $[10,11]$ are present some solutions for control the continuous casting process with fuzzy logic, but these solutions not realize a uniform repartition of the water flow in the secondary cooling stage and not work like an adaptive system. Other authors [12-14] use fuzzy logic and neural networks for detecting the cracks in crystallizing and control the primary water cooling stage.

The goal of intelligent system proposes by us is to improve the classical adjustment system, the introduction of some adaptive components in the adjustment loops and some overall predictions over the continuous casting machine. The fuzzy system can be adapted to all types of continuous casting plants, because it does not require major hardware changes, and is practically grafted into the existing structure of the continuous casting plant.

\section{The structure of the control system}

In Fig. 1 we describe the modern pattern of the control system of the continuous casting. The neural network of crack detection delivers the logic signal " 1 " at the moment when a primary crack was detected; otherwise the output signal is " 0 ". This information is used accordingly by a fuzzy decision system (FC) with two bases of rules, which analyze a set of data acquired from the process, triggering changes of cooling water flow rate $(\Delta v)$ and of casting speed set points $(\Delta q)$.

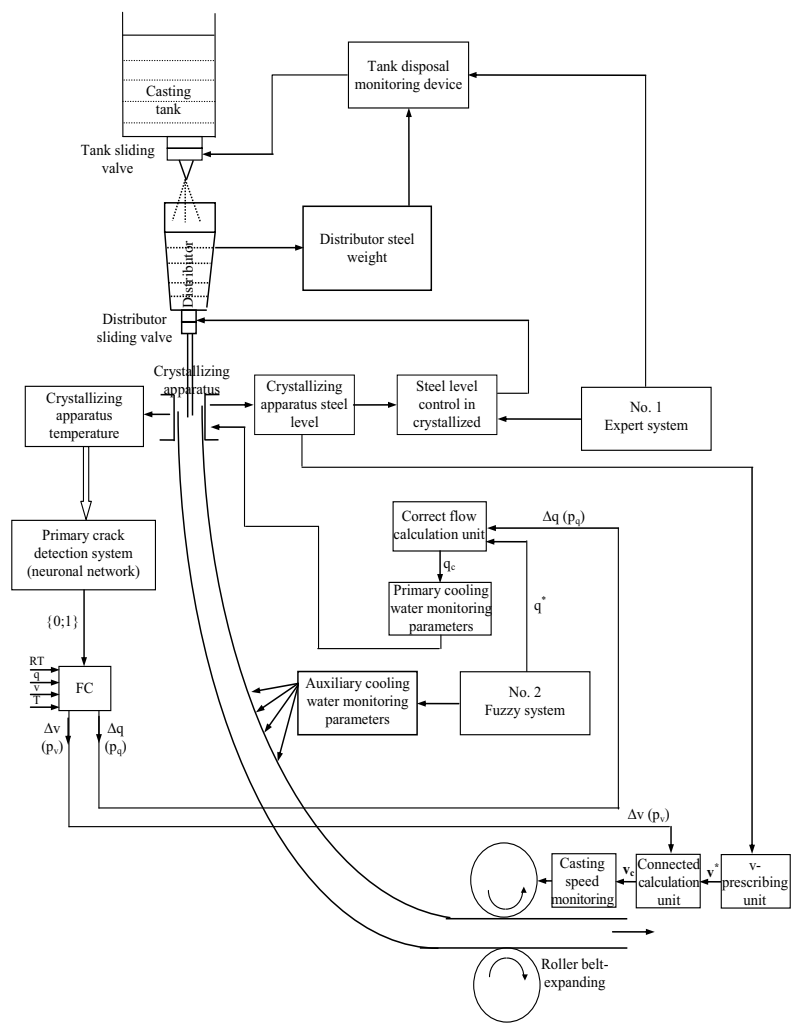

Figure 1. The structure of the control system.
Except for the technological risk (RT), which is set by the technological engineer, the other variables used as input data by the $\mathrm{FC}$ are received from the sensors already existing in the plant. The fuzzy decision system (FC) operates with two different rule bases, selected according to the signal ( 0 or 1$)$ received from the neural network for the detection of cracks [13]. Fig. 1 shows that the two FC outputs (the casting speed correction $\Delta_{\mathrm{v}}$ and the flow rate one $\Delta_{\mathrm{q}}$ ) are applied to calculation blocks that give the needed value for the correction of casting speed $\left(\mathrm{v}_{\mathrm{c}}\right)$ and the flow $\operatorname{rate}\left(\mathrm{q}_{\mathrm{c}}\right)$, which, also are applied to the control systems of the respective parameters, existing in the plant. So, these values will replace the imposed values $\mathrm{v}^{*}$, respectively $\mathrm{q}^{*}$ (control system initial set points) of the existent control system for both closed loops.

\section{The control strategy proposed}

The secondary cooling system is divided into 3 subareas such as: area 1 or foot roller area located on the crystallizing exit, area 2 and area 3 , these areas are controlled individually. In general the sprayed water flow must decrease from the crystallizing in the direction of the cast. The sprayed water flows must be bigger in the superior side of the casting machine (area 0 and area1), for realizing the increasing of the semi-products crust and thus improve the resistance crust according to the effort. Lowering the cooling intensity in the casting direction must prevent that the surfaces temperature became too low in the straightening points. How we already presented the cooling is done using some valves by using some classical PID loops which adjust the water flow depending on reference measure. The goal of an intelligent system is to improve this classical adjustment system, the introduction of some adaptive components. Fig. 2 shows the block diagram of the proposed control strategy.

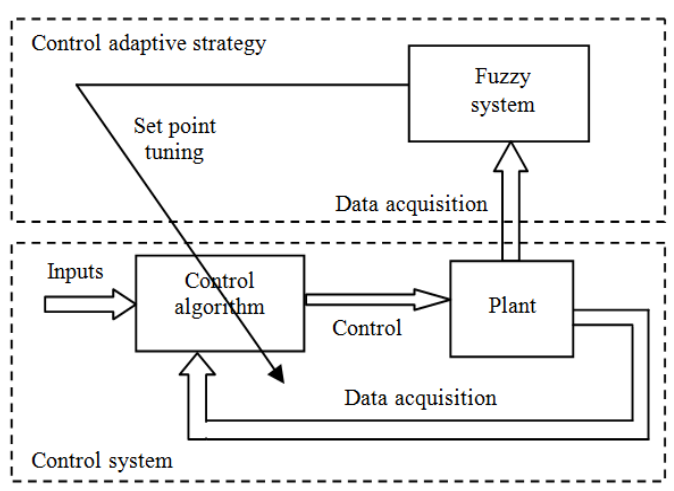

Figure 2. The block diagram of the control strategy.

From the technical point of view the changes made to the installation are not very important from the point of view of the execution and driving equipment, but are very important from the point of view of the control system, of the software which is used to control the system by introducing some fuzzy controllers, some predictive adaptive systems or by introducing some standard models.

From the technological point of view the monitoring system must be rethought so that the technologist know which the ranges of the casting deviation for each recipe 


\section{ICIEA 2016}

so he doesn't enter deviations that lead to different types of damage such as perforations of the semi-product, quality problems, problems related to the appearance of the casting surface and of the casting profile geometry.

The fuzzy system which will be created must also take into consideration the distance between the rings and the length of the curved wire. The installations geometry will reveal for a certain speed and casting recipe the needed quantity of the water to obtain the desired quality, this will make a spraying recipe which will give the quantity of water for every area, the values of the minimal flows needed for ensuring a minimal pressure in this areas ( the minimal pressure for this areas is 2 bar) and implicitly the information needed to create the fuzzy systems rules which may take the needed measures in case the waters pressure is too low or too high, pressure which must be assured by a corresponding flow.

The classical systems are limited to a rigid repartition of the water flow in each area such as: $40 \%$ on the first area, $40 \%$ on the second area, and $20 \%$ on the third area.

An intelligent system has the capacity of eliminating this nowadays systems disadvantage by having the possibility of modifying in real time the water flow repartition for each area taking into consideration what happens in the installation.

\section{Design of fuzzy controller}

We will create a fuzzy controller having 3 inputs: speed, temperature and water flow and 1 output: Flow correction. Matlab design of fuzzy controller is present in Fig. 3.

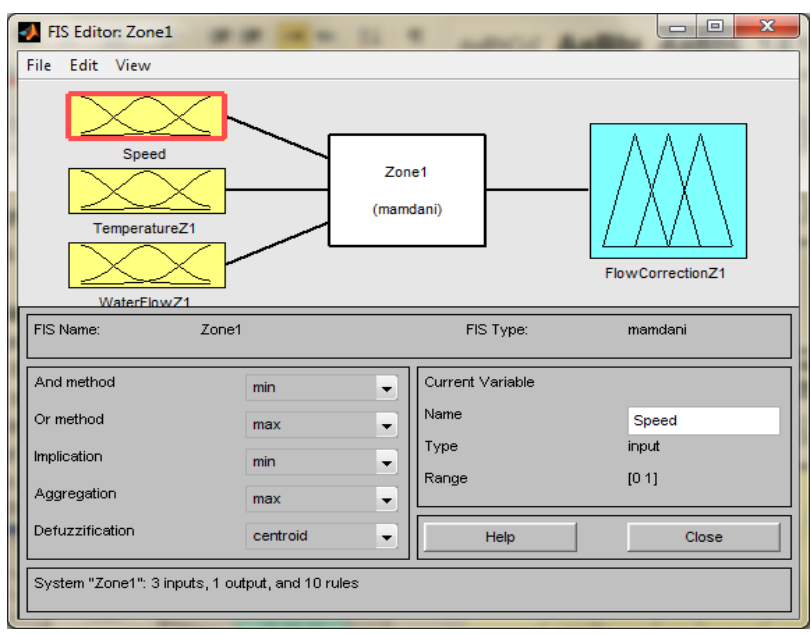

Figure 3. Block diagram of the fuzzy controller.

\section{Input information}

1) Speed (casting speed, $[\mathrm{m} / \mathrm{min}]$ )

Number of states: 5

\begin{tabular}{|c|c|c|}
\hline States & $\begin{array}{c}\text { Real domain } \\
{[\mathbf{m} / \mathbf{m i n}]}\end{array}$ & $\begin{array}{c}\text { Standardized } \\
\text { values domain }\end{array}$ \\
\hline Very small & $0.7-0.8$ & $0-0.2$ \\
\hline Small & $0.8-0.9$ & $0.2-0.4$ \\
\hline Medium & $0.9-1.0$ & $0.4-0.6$ \\
\hline High & $1.0-1.1$ & $0.6-0.8$ \\
\hline Very high & $1.1-1.2$ & $0.8-1$ \\
\hline
\end{tabular}

2) Temperature (steel temperature $\left[{ }^{0} \mathrm{C}\right]$ )
Number of states: 3

\begin{tabular}{|l|c|c|}
\hline \multicolumn{1}{|c|}{ States } & $\begin{array}{c}\text { Real domain } \\
{\left[{ }^{\mathbf{0}} \mathbf{C}\right]}\end{array}$ & $\begin{array}{c}\text { Standardized } \\
\text { values domain }\end{array}$ \\
\hline Small & $410-610$ & $0-0.33$ \\
\hline Medium & $610-810$ & $0.33-0.66$ \\
\hline High & $810-1010$ & $0.66-1$ \\
\hline
\end{tabular}

3) Water Flow (water flow [1/min $]$ )

Number of states: 5

\begin{tabular}{|l|c|c|}
\hline \multicolumn{1}{|c|}{ States } & $\begin{array}{c}\text { Real domain } \\
{[\mathbf{l} / \mathbf{m i n}]}\end{array}$ & $\begin{array}{c}\text { Standardized } \\
\text { values domain }\end{array}$ \\
\hline Very small & $900-940$ & $0-0.2$ \\
\hline Small & $940-980$ & $0.2-0.4$ \\
\hline Medium & $980-1020$ & $0.4-0.6$ \\
\hline High & $1020-1060$ & $0.6-0.8$ \\
\hline Very high & $1060-1100$ & $0.8-1$ \\
\hline
\end{tabular}

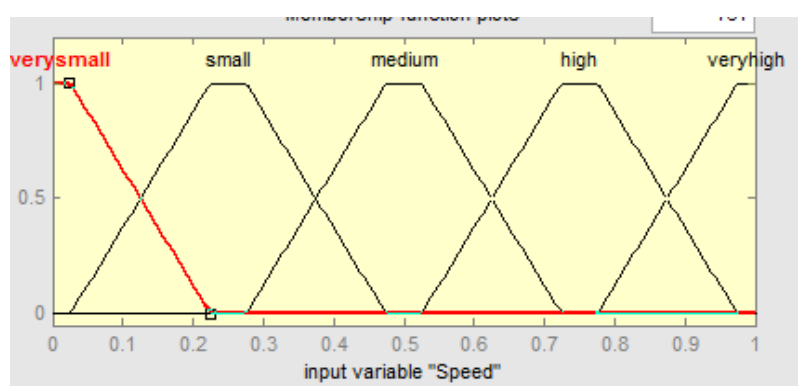

Figure 4(a). Membership function „Speed”.

In Figs. 4(a), 4(b) and 4(c) we describe the membership functions for the input sizes.

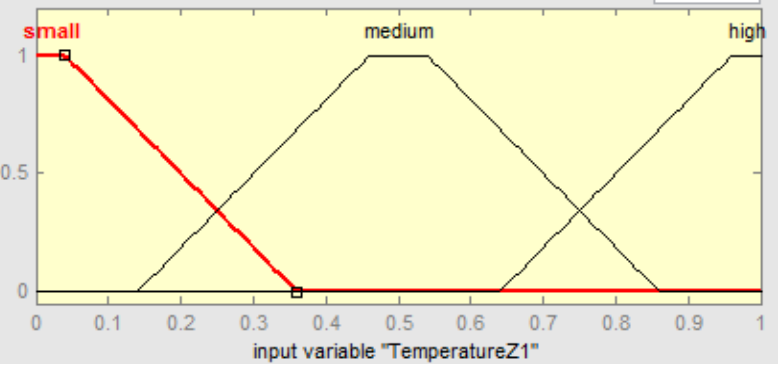

Figure 4(b). Membership function „Temperature”.

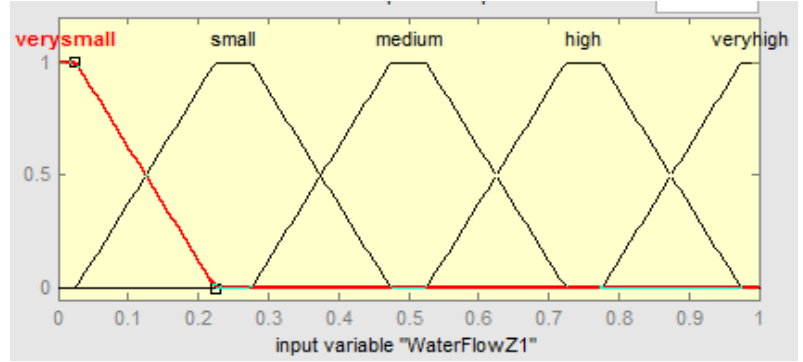

Figure 4(c). Membership function ,Water Flow”.

\section{Output information}

1) Flow Correction (water flow correction [1/min])

Number of states: 5

\begin{tabular}{|c|c|c|}
\hline States & $\begin{array}{c}\text { Real domain } \\
{[\%]}\end{array}$ & $\begin{array}{c}\text { Standardized } \\
\text { values domain }\end{array}$ \\
\hline Verysmall & $0-4$ & $0-0.2$ \\
\hline Small & $4-8$ & $0.2-0.4$ \\
\hline Medium & $8-12$ & $0.4-0.6$ \\
\hline High & $12-16$ & $0.6-0.8$ \\
\hline Veryhigh & $16-20$ & $0.8-1$ \\
\hline
\end{tabular}


In Fig. 4(d) we describe the membership functions for the output size.

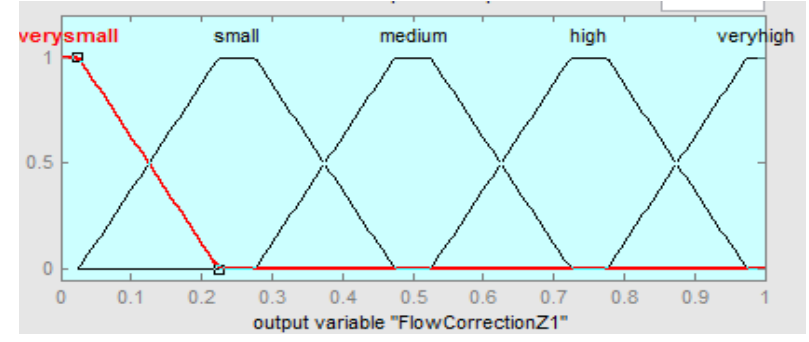

Figure 4(d). Membership function „Flow Correction”.

Table of inference for some of the rules is given below:

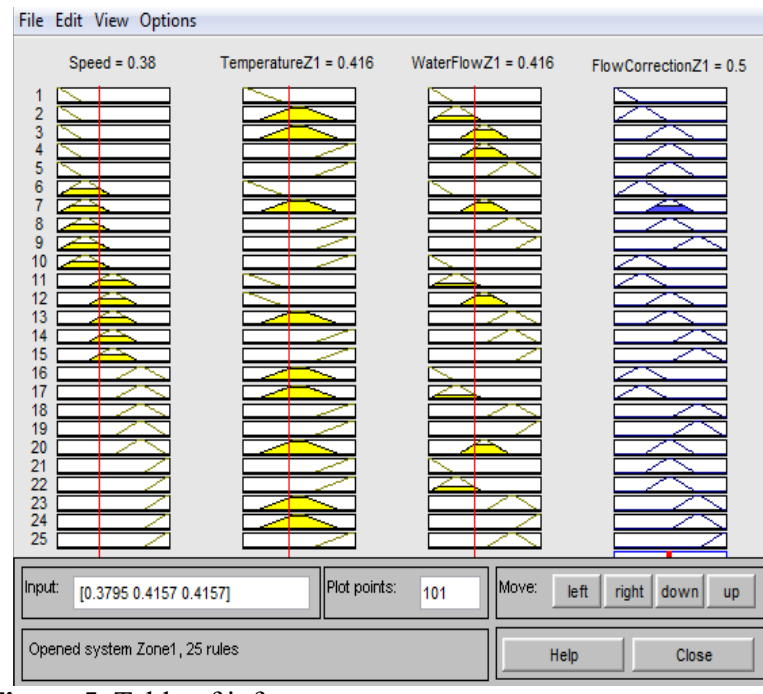

Figure 5. Table of inference.

Control surfaces obtained by simulations according to the block diagram from the Fig. 3 are listed below:

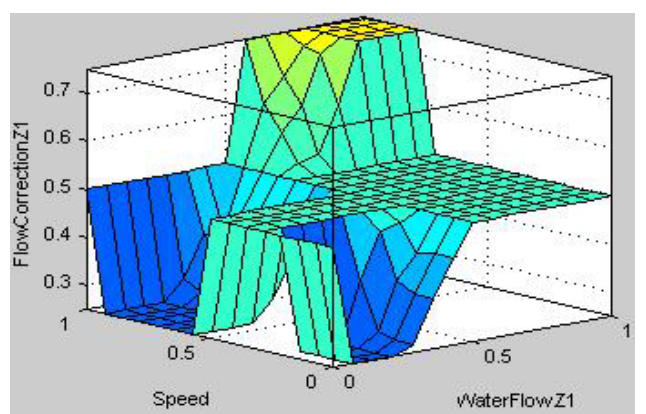

Figure 6. Control surface - Flow correction $=\mathrm{f}$ (speed, water flow).

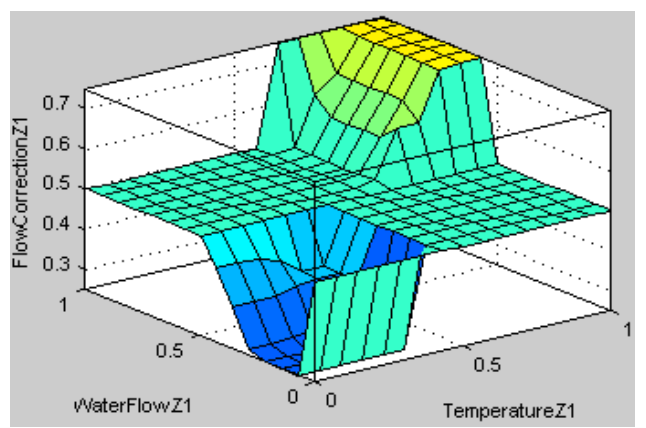

Figure 7. Control surface - Flow correction $=\mathrm{f}$ (water flow, temperature).

\section{Validation of the simulated system}

We have applied two different sets of data measured during the current process and stored into tables in case of validation of the simulated system operation, and for the input crack detection neural network. One of the sets refers to the situation when there are no cracks and the other one in case cracks occur.

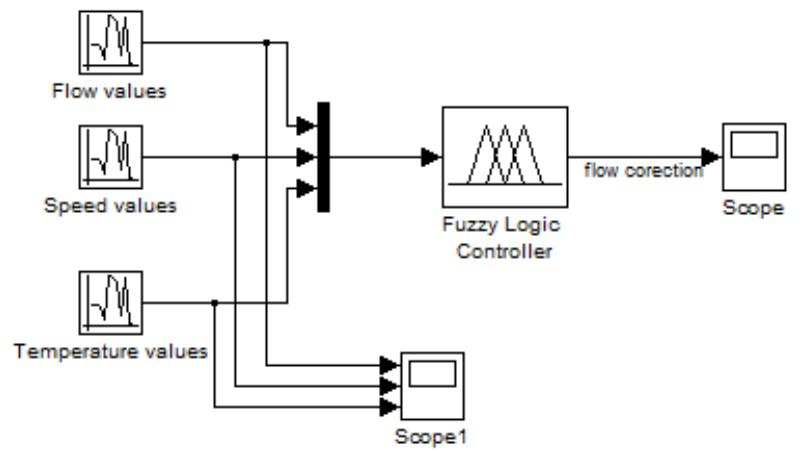

Figure 8. Simulink implementation of the fuzzy controller.

At the fuzzy controller input, there are several input values (flow, speed, temperature). These values are described in Fig. 8:

a) Time variation (120 seconds) of fuzzy controller input values;

b) Flow correction and new flow values.

Fig. 9 describes the situation during the first 30 simulation seconds, when both the cooling water flow and casting speed are low, the temperature is high. Speed correction is very low, hence required casting speed is almost unchanged.
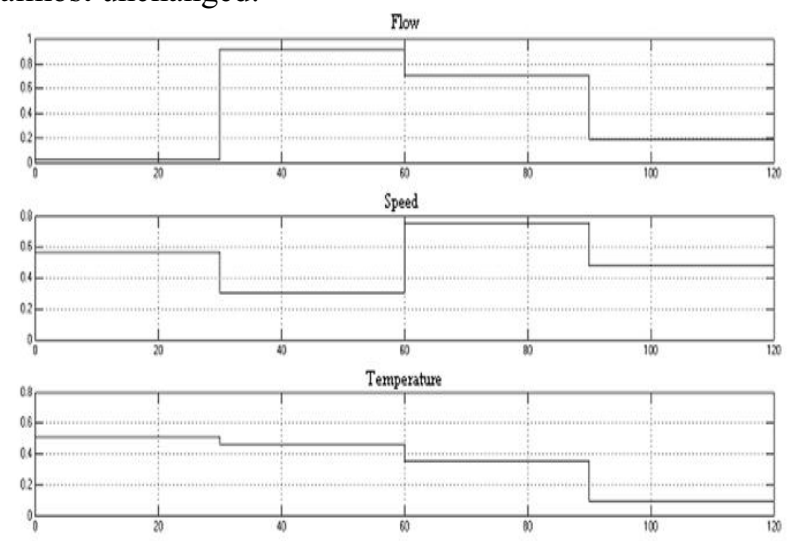

a) RG-F Input Data
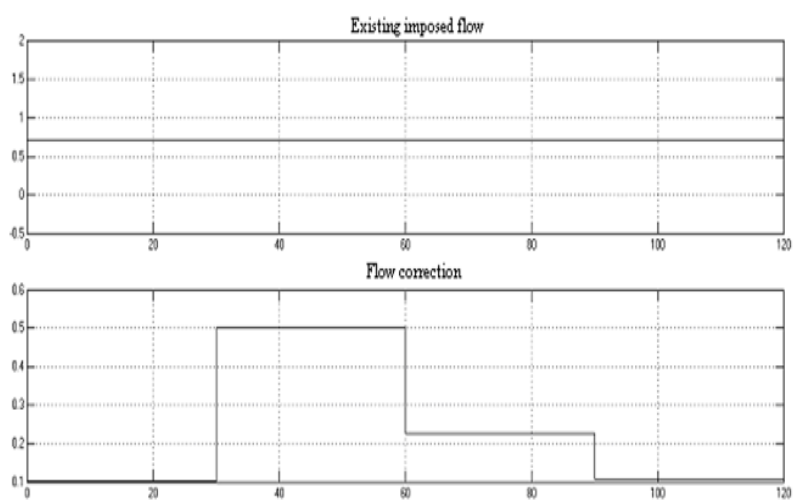

b) Output data - flow

Figure 9. Fuzzy controller validations. 
During the whole time, cooling water flow increases significantly. We can see that the other two simulation rounds are similar. When analyzing all cases described in Fig. 9 we draw the following conclusions: fuzzy controller analyzes the input values and elaborates water flow corrections, according to the base sets; fore-writing block corrects all required values for flow, according fuzzy controller outputs. By simulating in Simulink, we have proved that all solutions are correct. Such simulation is made for performing a check out of the fuzzy system. During operation, all size values do not change so fast, hence some input values combinations are not that predictable. Once the system is implemented, the rules referring to such situations could be eliminated.

\section{Conclusions}

The main target of this paper was to develop an intelligent control system of the continuous casting process in the secondary cooling area capable of eliminating the surface flaws and casting rejects, to increase the quality of continuously cast products and implicitly to increase the productivity of the installation by controlling the water flow rate in the secondary cooling area. In order to achieve this objective, a solution was developed, meant to control in real time the distribution of the water flow rate according to the real situation in the installation and working as an adaptive system. The system was tasted by simulation and the results show us that the control strategy proposed is a very efficient method for the water flow control in continuous casting.

\section{Acknowledgment}

This work was supported by a grant of the Romanian National Authority for Scientific Research and Innovation, CNCS - UEFISCDI, project number PN-IIRU-TE-2014-4-1788.

\section{References}

1. E. Ardelean, et al., Simulation of continuous cast steel product solidification, Revista de Metalurgia 43 (3), pp.181-187, (2007)

2. V. A. Efimov, Casting and Crystallizing of Steel, Ed. Technical, Bucuresti, (1986).
3. B. G. Thomas, J. T. Parkman, Simulation of thermal mechanical behavior during initial solidification, Internat. Conf. On Thermomechanical Processing of Steel and Other Materials, Wollongong, Australia, TMS, (1997)

4. B. G. Thomas, Modeling of the continuous casting of steel-past, present and future, 59th Electric Furnance Conference, Phoenix, AZ, (2001)

5. G. O. Tirian, C. Pinca, C. Abrudean, C. Chioncel, Analytical identification, simulation and analysis of steel solidification process in crystallizer, Annals of University "Eftimie Murgu" Resita, XVIII, Nr. 1, (2010)

6. M. Janik, H. Dyja, Modeling of three-dimensional temperature field inside the mould during continuous casting of steel, Journal of Materials Processing Technology, Volumes 157-158, pp. 177-182, (2004)

7. J. Stetina, L. Klimes, T. Mauder, New challenges in modelling and secondary cooling control of continuous steel casting, MATEC\& $2^{\text {nd }} E S T E A D^{2015}$, Dusseldorf, Germany, pp. 1-8, (2015)

8. B. Kiflie, D. Alemu, Thermal analysis of continuous casting process, ESME 5th Annual Conference on Manufacturing \&Process Industry, Addis Ababa University, Ethiopia, (2000)

9. C. Furtmüller, L. D. Re, Control issues in the continuous casting of steel, Proceedings $17^{\text {th }}$ World Congress Int. Feder. Autom. Cont., Seoul, Korea, pp. 700-7005, (2008)

10. L. Mugwagwa, et al. Design of a fuzzy controller for the continuous casting process, International Journal of Engineering Science and Technology, 5, 1, (2013)

11. T. Mauder, J. Stetina, M. Masarik, On-line fuzzy regulatorfor continuous casting process, Metal 2013, Brno, Czech Republic, EU, (2013)

12. A. K. Bhattacharya, et al., Fuzzy diagnostics system for breackout prevention in continuous casting of steel, Intelligent Control and Automation, WCICA 2004, pp. 3141-3145, 4, (2004)

13. G. O. Tirian, I. Filip, G. Prostean, Adaptive control system for continuous steel casting based on neural networks and fuzzy logic, Neurocomputing, pp. 236-245, 125, (2014)

14. G. O Tirian, et al., Fuzzy system for implementing the cracks control during the continuous casting, Proceedings of the 20th International Symposium, 20, 1, pp. 1661-1662, Austria, (2009) 